\title{
Aaron Swartz's JSTOR code : \\ keepgrabbing.py. Further research notes.
}

Camille Akmut

December 30, 2019

\begin{abstract}
Technical as well as sociological, legal and historical characteristics of a critical program.
\end{abstract}




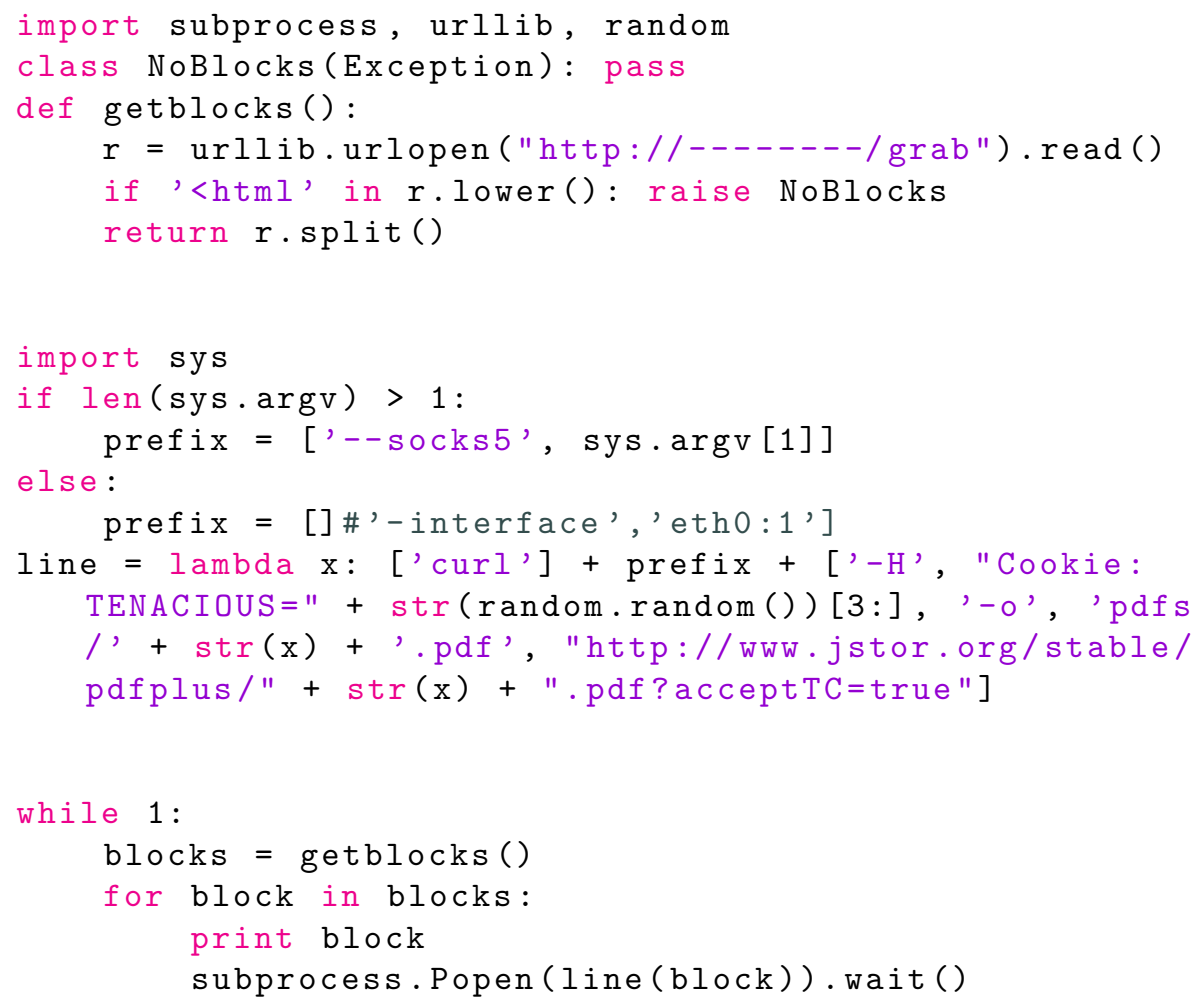

Figure 1: keepgrabbing.py 


\section{Aaron Swartz JSTOR code}

keepgrabbing.py further research notes

$29-12-19$

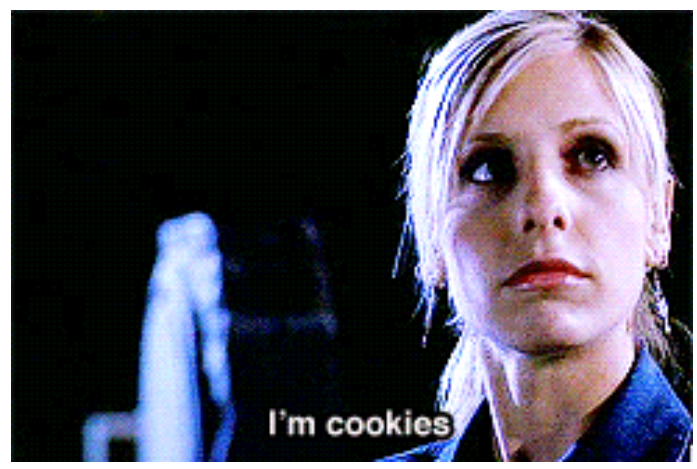

\section{context}

"Swartz's ... laptop contained a software program named "keepgrabbing.py," which was designed to download .pdf files ... from JSTOR"

(USA v. Swartz)

\section{overall architecture}

(The program can be read by anyone with 3-4 years of programming. I'll do my very best to explain how it works, but I can't guarantee I won't make any mistakes.)

The whole program is based around cur $\mathrm{l}$, a utility to download files from the Internet/Web, from the command-line.

"a tool to transfer data from or to a server", its man(ual) page tells us.

This is of particular significance since the whole point of the program was to download .PDF files (i.e. academic papers, a lot of them publicly funded) massively from JSTOR servers.

"The command is designed to work without user interaction." the man page accordingly goes on.

Alternatively, Swartz could have used wget . 
(The reason he used curl is perhaps that the Developer Tools section found in browsers give translations for GET requests in curl format but not in wget's e.g. Firefox.)

So, the general architecture :

1. getblocks() generates a list of research paper titles or more precisely their nomenclature (whatever they use at JSTOR)

e.g. :

\section{On_the_Landau-Siegel_Zeros_Conjecture regions_and_social_groups \\ etc.}

or perhaps, given a url from https://server.org/pdf/0000.4000.pdf :

0000.4000

0505.1000

etc.

whatever.

This is the first block of code.

The output of which is associated with the variable "blocks" at the end. Each individual entry is accessed via "block".

1. the lambda function takes this as input

2. curl is executed on all papers, with some extra parameters presumably to make sure the server will cooperate and not throw up errors (header, cookies, ...)

\section{the lambda function}

As a functional programmer, I'm particularly drawn to the lambda function and it's arguably the heart of the program.

line = lambda $x:$ ['curl'] + prefix + ['-H', "Cookie: TENACIOUS=" + str(random. random())[3:], '-o', 'pdfs/' + str(x) + '.pdf', "http://www.jstor.org/stable/p dfplus/" + str(x) + ".pdf?acceptTC=true"] 
This looks like a lot, but the tools that we have at our disposal are always the same, and very effective too : simplify, experiment.

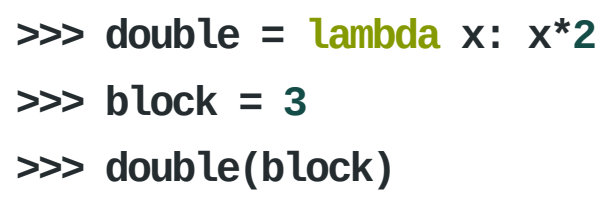

6

and further :

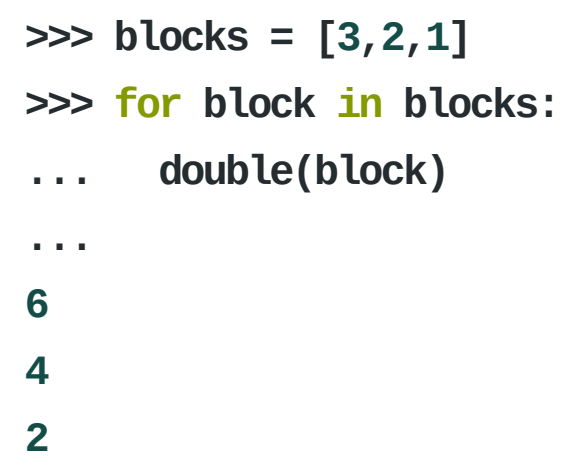

So what is going on here?

We must jump around the program to make sense of it, it is not a novel.

\section{subprocess. Popen( Line(block)) . wait()}

Here, line is passed "block". This is our " $x$ " in "lambda x"! At least this is my best understanding.

Let's go back to the lambda line and erase all of the extra stuff that's fundamentally not needed:

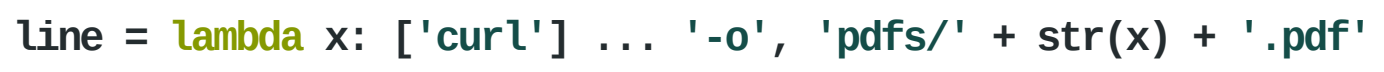

This is just Aaron telling curl : please download all of the articles inside the local folder "pdfs" and label them nicely.

e.g.

.$-- /$ pdfs

- On_the_Landau-Siegel_Zeros_Conjecture.pdf

- regions_and_social_groups.pdf 
etc.

This is the core of it

line = lambda $x:$ ['curl'] ... "http://www.jstor.org/stable/pdfplus/" + str(x)

+ ".pdf..."]

translated later as the familiar command line command :

\$ curl (-0) http://wWw.jstor.org/stable/pdfplus/On_the_Landau-Siegel_Zeros_Con jecture.pdf

(minus the extra parameters)

The "prefix" bit is just Aaron passing an additional parameter to curl, "-socks5".

As with everything else, it can be looked up in this utility's, curl's manual :

man curl | grep "socks5"

returns :

- -socks5 <host [:port]>

He was using a proxy. (?)

\section{getblocks()}

It's hard to say much about this, because one part has been redacted.

But “http://-------/grab" was definitely able to generate a list of all of JSTOR's articles.

〉 "article1 article2".split()

['article1', 'article2']

the Python built-in function split() does stuff like that. 


\section{References}

- 2019. "A User-Defined Web. And, on Systems modification in general." Buffy. S07E22. (Final episode : "Cookie dough speech") 